Apidologie 1980, 11 (2), 131-141.

\title{
ZUR METHODIK DER PRÜFUNG VON CHEMOTHERAPEUTIKA ZUR BEKÄMPFUNG DER VARROATOSE DER HONIGBIENE
}

\author{
Méthode de test des substances chimiothérapeutiques \\ dans la lutte contre la varroose chez l'abeille domestique
}

\author{
Wolfgang RITTER \\ Fachbereich Biologie d.J.W.Goethe-Universität Frankfurt \\ Institut für Bienenkunde \\ Im Rothkopf 5, D-6370 Oberursel/Ts.
}

\section{ZUSAMMENFASSUNG}

Zur Untersuchung in der Varroatose-Bekämpfung verwendbarer Wirkstoffe wurde ein Verfahren entwickelt, das in stufenweisen Versuchsansätzen die gleichzeitige Bestimmung der $L D_{s_{0}}$ für Bienen und Milben erlaubt. Im Labor wird der geeignete Wirkstoff, seine Dosierung, Formulierung und Applikation durch bienentoxikologische Versuche mit jeweils 100 Bienen und durch milbentoxikologische Versuche mit jeweils 1500 bis 2000 Bienen ausgewählt. In Freiland- und Feldversuchen wird die Wirkung in Bienenvölkern überprüft.

Die behandelten Bienen werden zur quantitativen Gewinnung aller auf ihnen verbliebenen Milben in Waschbenzin ausgeschüttelt.

\section{SUMMARY \\ METHOD FOR TESTING CHEMOTHERAPEUTICAL SUBSTANCES USED IN CONTROLLING $V A R R O A$ DISEASE}

A method was developed for testing the substances used in controlling Varroa disease in different steps to examine $\mathrm{LD}_{50}$ both in bees and Varroa mites. The drugs are first tested in the laboratory, on a small number of caged bees (approximately 100 bees in each cage) and then on an artificial swarm of about 2,000 bees, to find out the best drug, dose and manner of application. Fieldtests are made to examine results in colonies.

The treated bees were shaken in gasoline, in order to find the number of mites still on their body. 


\section{EINLEITUNG}

Die bis vor kurzem bekannten Medikamente zur Behandlung der Varroatose zeigten nur eine unzureichende Wirkung. Der Erprobung neuer bisher nicht untersuchter akarizider Wirkstoffe kommt daher eine besondere Bedeutung zu.

Die Zulassung eines Mittels als Medikament setzt eine eingehende biologische Prüfung voraus, die über verschiedene Stationen verläuft (Abb. 1). Vorher wird die

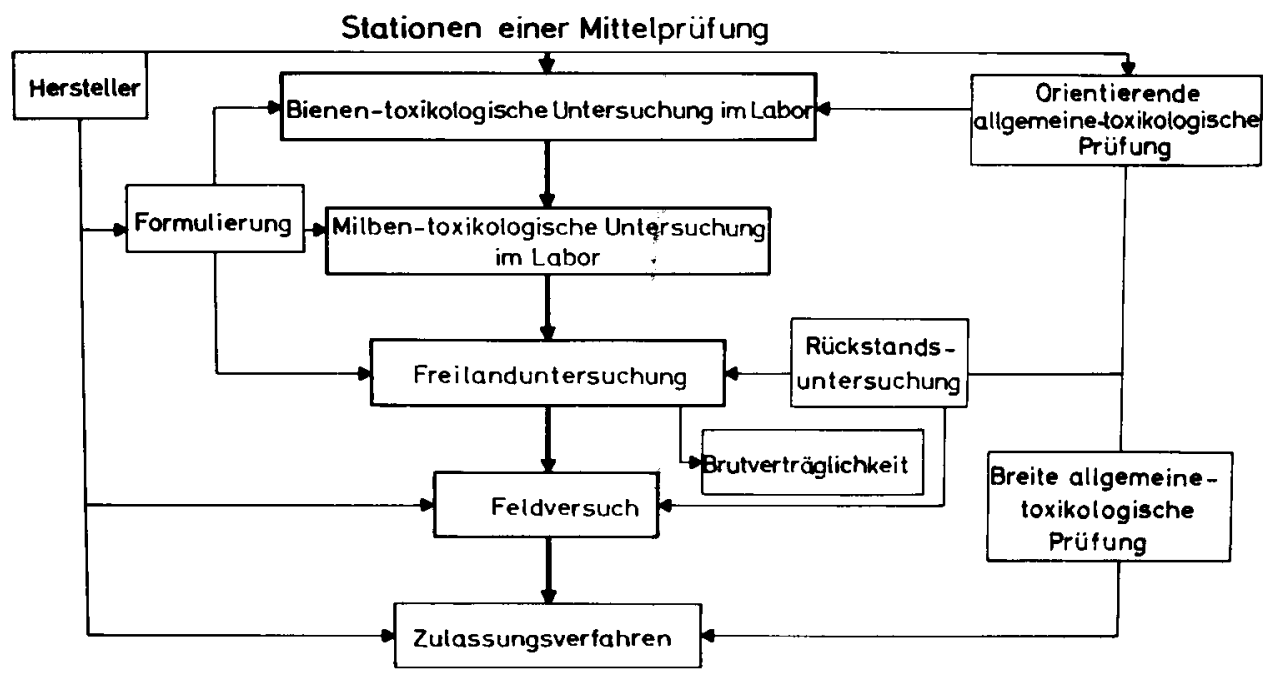

Aв8. 1. - Stationen einer Mittelprüfung.

FIG. 1. - Étapes dans le travail de test d'une substance.

Auswahl der zu prüfenden Substanzen aus " nicht bienengefährlichen " Pflanzenschutzmitteln, Neuentwicklungen der Industrie und anderen organischen Verbindungen getroffen. Der biologische Test beginnt im Labor mit der bienen- und milbentoxikologischen Untersuchung, an die sich Freilandtests anschliessen. Der Präparate-Hersteller unterstützt die Arbeit durch eventuelle Änderungen der Formulierung und bei Neuentwicklungen durch orientierende toxikologische Untersuchungen an Vertebraten. Hat ein Präparat bis zu dieser Station die Mittelprüfung erfolgreich durchlaufen, so gibt der Hersteller den Auftrag für die klinische Erprobung in Feldversuchen, die in Zusammenarbeit mit den Veterinärbehörden durchgeführt werden. Rückstandsuntersuchungen und breite toxikologische Untersuchungen leiten das Zulassungsverfahren ein. Die bei der biologischen Prüfung angewandte Methode wird im folgenden ausführlich dargestellt.

\section{BIENENTOXIKOLOGISCHE UNTERSUCHUNG}

Zur bienentoxikologischen Untersuchung werden jeweils etwa 100 Bienen in kleine Käfige gefüllt (Abb. 2). Die Fütterung erfolgt mit Zuckerwassser im Verhältnis 


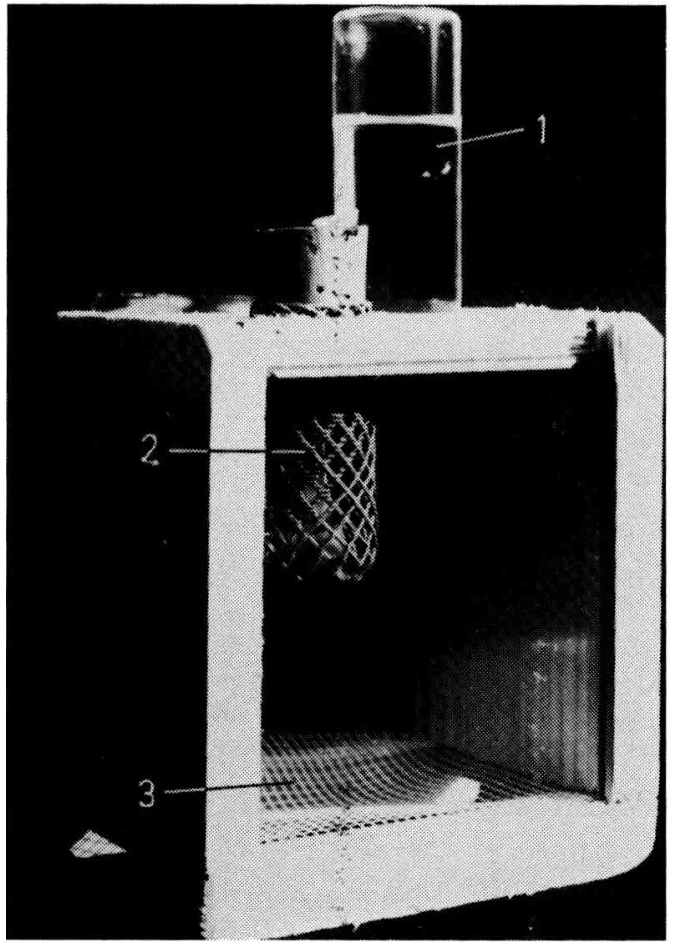

AвB. 2. - Käfig für die bienentoxikologische Untersuchung.

1 : Futtergefäss $\quad 2$ : Korb für Verdunstungsmittel $\quad 3:$ Gitterboden. .

FIG. 2. - Cage pour l'étude de la toxicité vis-à-vis de l'abeille.

1 : nourrisseur 2 : corbeille pour le produit volatil $3:$ fond grillagé.

$1: 1$. Die gefüllten Käfige werden in einem Raum mit einer konstanten Temperatur von $20^{\circ} \mathrm{C}\left( \pm 1^{\circ} \mathrm{C}\right)$ und einer relativen Luftfeuchtigkeit von $55 \%( \pm 5 \%)$ aufgestellt. Da der Toxiditätsgrad von Wirkstoffen keine absolute, sondern relative Grösse ist, die von der Temperatur, Luftfeuchtigkeit und Wirkzeit abhängt, müssen bei den bienen- und milbentoxikologischen Untersuchungen diese Versuchsbedingungen immer eingehalten werden.

Nach 24 Stunden, in denen sich die Bienen akklimatisieren können, erfolgt die Behandlung. Zur Prüfung von Spritzmitteln werden die Bienen durch den Gitterboden mit $1 \mathrm{ml}$ einer Suspension oder Emulsion fẹin besprüht (Sprüheinrichtung : Spray-Gun, Desaga GmbH). Als Lösungsmittel werden je nach der Art des Mittels Wasser, Aceton oder Isopropylalkohol verwendet. Verdunstungspräparate werden in kleinen Glasgefässen $(2 \mathrm{ml}$ Inhalt) mit einem $1 \mathrm{~cm}$ überstehenden Papierdocht in die Käfige gestellt. Ein Korb (Lockenwickler) verhindert den direkten Kontakt der Bienen mit dem Behandlungsmittel. Die verdunstete Flüssigkeitsmenge wird durch Abwiegen der Gefässe vor und nach dem Versuch bestimmt. Systemisch wirkende Präparate werden in verschiedenen Dosen dem Zuckerwasser zugesetzt und an die Bienen verfüttert. Der Futterverbrauch wird am Ende des Versuchs bestimmt. Zur Prüfung von Räuchermitteln sind die Kleinschwärme nur bedingt geeignet, da die Mittel an den 
Glasflächen der Käfige niederschlagen und somit eine genaue Applikation nicht möglich ist.

Zunächst wird der ungefähre Bereich der Grenzdosis für Bienen durch Tastversuche bestimmt. Innerhalb dieses Bereichs werden verschiedene möglichst geometrisch abgestufte Dosen getestet und anhand der Bienenmortalität nach 24 Stunden die Dosis letalis minima $\left(\mathrm{LD}_{50}\right)$ bestimmt. Gleichzeitig können die auf die Unterlage gefallenen Milben einen ersten Hinweis auf die akarizide Wirkung des Mittels geben. In einzelnen Fällen werden die Versuche bis zu 70 Stunden verlängert. Im allgemeinen werden für jede Substanz Versuche mit 4 Dosierungen und 3 Wiederholungen angesetzt. Jede Versuchsreihe enthält 2 bis 4 Kontrollen. Da der Wirkstoff nur selten in reiner Form verwendet werden kann, werden Versuche mit verschiedenen Formulierungen und Lösungsmitteln einbezogen. Bestimmung des Restmilbenbefalls erfolgt nach der unter $5 b$ ) beschriebenen Methode.

\section{MILBENTOXIKOLOGISCHE UNTERSUCHUNG}

Aus befallenen Völkern werden grössere Einheiten mit 1500 bis 2000 Bienen zusammengestellt, eine Art Kunstschwarm, jedoch ohne Königin. Wir halten es für richtiger, die Prüfung mit natürlich infizierten Bienen vorzunehmen, als nach LANGE (1976) 50 Milben auf 50 Bienen zu setzen. Die Kunstschwarmkästen (Abb. 3) sind an der Unterseite mit einer Gaze (2,6 mm Maschenweite) versehen, so dass die Milben ungehindert hindurchfallen und nicht auf die Bienen zurückgelangen können. Die Gaze an den Seitenflächen ermöglicht eine direkte Behandlung des Schwarmes.

Die Aufstellung der Schwarmkästen und die Behandlung der Bienen entspricht weitgehend der bienentoxikologischen Untersuchung (s. Abschnitt 1). Die milbentoxikologische Untersuchung erfolgt in dem für Bienen ungefährlichen Dosisbereich. Zusätzlich wird die verabreichte Menge des Behandlungsmittels um den Faktor 5 verringert. So werden $4 \mathrm{ml}$ der Suspension und Emulsion auf die Bienen gesprüht, d.h. $0,002 \mathrm{ml}$ statt $0,01 \mathrm{ml}$ Mittel pro Biene.

Zur Prüfung von Verdunstungs- und Räuchermitteln werden jeweils 2 Schwarmkästen in eine leere Beute gestellt. Das Verdunstungsmittel wird in einer Flasche mit einem $3 \mathrm{~cm}$ langen Papierdocht zwischen beide Kästen gestellt. Räuchermittel werden entweder über glühender Holzkohle im Rauchapparat verbrannt und durch das Flugloch in die Beute eingebracht oder die Substanz auf Papierstreifen bzw. in Metalldosen in der Beute verraucht.

Für jede Substanz werden 3 Dosierungen mit jeweils 2 Schwärmen und 2 Kontrollen mit dem Lösungsmittel bzw. ohne Behandlung angesetzt. Nach 24 Stunden wird anhand der Milbenmortalität die Dosis letalis minima $\left(\mathrm{LD}_{50}\right)$ bestimmt. In einzelnen Fällen kann die Versuchszeit zur Überprüfung einer verzögerten Wirkung verlängert werden. Neben der milbentoxikologischen Prüfung kann besonders bei 
Spritzmitteln eine weitere Überprüfung der Bienenmortalität erfolgen. Der Restmilbenbefall wird nach der unter Diagnose beschriebenen Methode (Abschnitt 5 b) bestimmt.

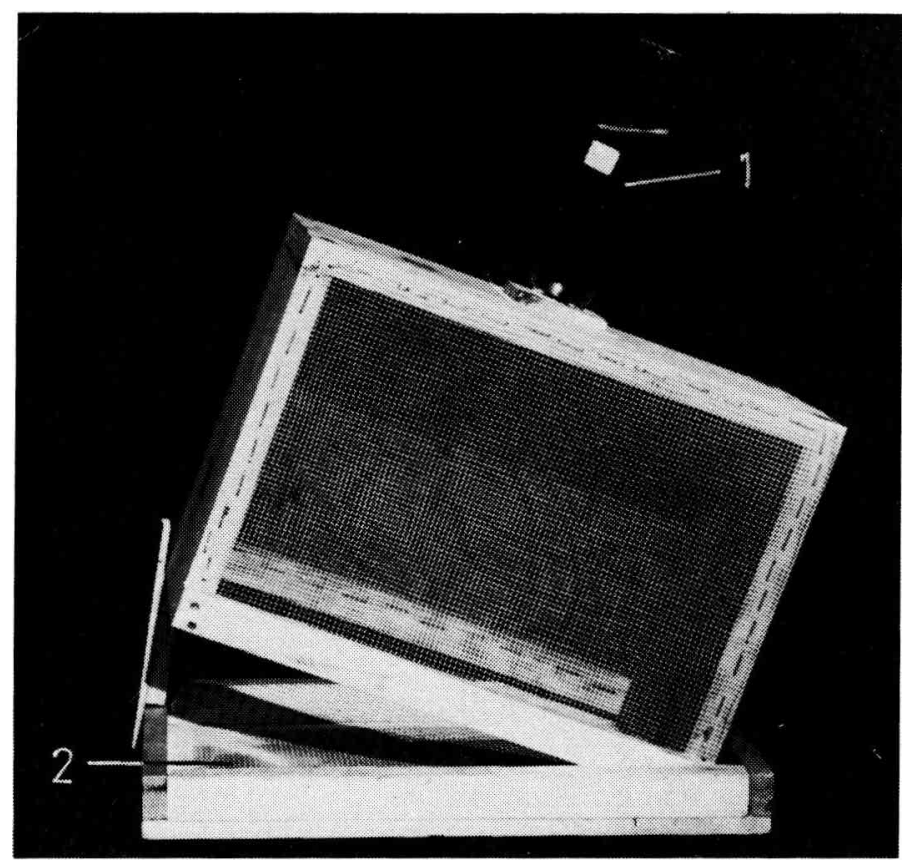

Aвв. 3. - Schwarmkasten für die milbentoxikologische Untersuchung (Aufgeklappt).

$$
1 \text { : Futtergefäss } 2 \text { : Gitterboden. }
$$

FIG. 3. - Caisse à essaim pour l'étude de la toxicité $v i s-\grave{a}$-vis des acariens (bords relevés). 1 : nourrisseur 2 : fond grillagé.

\section{FREILANDVERSUCHE}

Die Untersuchungen im Freiland werden zunächst an Völkern in Ablegerstärke mit 4 besetzten Waben und später in Vollvölkern durchgeführt. Die Völker enthalten entweder Brut mit verschiedenen Entwicklungsstadien oder ihnen wird vor der Behandlung sämtliche Brut entnommen.

Bei Spritzmitteln werden die Völker mehrmals im Abstand von mindestens 3 Tagen behandelt. Jeweils $10 \mathrm{ml}$ der Suspension bzw. Emulsion werden mit einem Drucksprüher (Gloria 48 002) auf jede von den Bienen besetzte Wabenseite gesprüht. Hierzu müssen die Waben herausgenommen oder verschoben werden. Verdunstungspräparate werden in einer Flasche mit variablem Docht in die Mitte einer leeren Zarge über den Bienensitz oder seitlich neben die Waben gestellt. Die verdunstete Flüssigkeitsmenge wird mit Hilfe eines Messstabes bestimmt. 
Räuchermittel werden wie bei der milbentoxikologischen Untersuchung (Abschnitt 2) angewandt. Das Flugloch muss jedoch meist für einige Minuten nach der Behandlung geschlossen werden, um das Ausziehen der Bienen zu verhindern. Die Versuchsansätze werden 4 bis 5 mal wiederholt.

Die abgestorbenen Bienen und Milben werden im Abstand von 2 Tagen (s. Abschnitt 5 a) und der Brutstand wöchentlich kontrolliert. Honigproben werden nach verschieden langer Versuchsdauer für erste Rückstandsuntersuchungen dem Volk entnommen. Die Bestimmung des Restmilbenbefalls erfolgt nach der unter 5 (Abschnitt $5 b$ ) beschriebenen Methode.

Der Test eines Präparates bis zum Ende der Freilandversuche dauert etwa 2 Monate. Bei ungünstigen Ergebnissen wird die Prüfung bereits in der ersten oder zweiten Laborphase abgebrochen. Dieses Verfahren wurde entwickelt, um eine möglichst grosse Zahl von Daten bei sparsamem Einsatz von Mitteln und Arbeitszeit zu erhalten. Der Materialaufwand für die Prüfung eines Mittels ist mit 2 Völkern für den Labortest und bis zu 7 Völkern für die Freilanduntersuchung recht gross, jedoch für eine exakte Mittelprüfung unerlässlich.

\section{FELDVERSUCHE}

Die letzte Station der Mittelprüfung bilden die Feldversuche. Im allgemeinen werden sie in einem geschlossenen Gebiet mit 100 bis 200 Völkern durchgeführt. Zur Brutzeit bleibt ein Grossteil der Milben unter den Zelldeckeln für die Behandlung unerreichbar. Behandlungen im zeitigen Frühjahr und im Spätherbst sind aus Witterungsgründen nur schwer durchführbar. Besonders beim Einsatz von Spritz- und Räuchermitteln müssen die Völker durch Entnahme der Brut oder durch dreiwöchiges Sperren der Königin brutfrei gemacht werden. Verdunstungspräparate können entweder im brutfreien Volk oder über eine Zeit von mehr als drei Wochen angewandt werden. In diesem Zeitraum wird jede Brutzelle auch im offenen Zustand erreicht. Der Behandlungserfolg wird an einer Stichprobe von etwa $10 \%$ der behandelten Völker überprüft.

\section{DIAGNOSE}

a) Bestimmung der Milbenmortalität im Bienenstock

Die nach der Behandlung im Bienenvolk abfallenden Milben werden auf einer hellen Papier- oder Kunststoffeinlage gesammelt, die zuvor in allen Versuchsvölkern eingeschoben wurde. Über der Einlage wird im Abstand von mindestens $6 \mathrm{~mm}$ eine Gaze mit mindestens 2,6 mm Maschenweite angebracht. Hierdurch wird verhindert, dass die Milben auf die Bienen zurückgelangen können oder von den Bienen 
hinausgetragen werden. Werden die Milben durch die Behandlung nur vorübergehend betäubt, so muss die Einlage zusätzlich mit einem Haftmittel (z. B. Vaseline oder technisches Fett) bestrichen werden.

b) Bestimmung des Restmilbenbefalls des behandelten Bienenvolkes

Wesentlich für die Beurteilung der Labor-, Freiland- und Feldversuche ist die Bestimmung der nach der Behandlung auf den Bienen und in der eventuell vorhandenen Brut verbliebenen Milben.

- Restmilbenbefall der Brut

Die gedeckelten Brutwaben werden nach Völkern getrennt in milbendicht verschlossene Behälter gestellt und im Brutschrank bei $34^{\circ} \mathrm{C}$ und $70 \%$ rel. Luftfeuchtigkeit zum Schlüpfen gebracht. Die ausgeschlüpften Bienen werden abgetötet und wie adulte Bienen untersucht. Bei geringen Brutmengen oder bei der Untersuchung einer Brutprobe (mindestens 300 Zellen) können die Zellen auch einzeln untersucht werden.

\section{- Restmilbenbefall der adulten Bienen}

Zunächst wurde der Restmilbenbefall durch die Nachbehandlung mit einem Standardpräparat bestimmt (RITTER, 1978 b). Als Standardpräparat wurde Dicofol (Kelthane-Hoechst) verwendet, das sich als gut wirksam gegen Milben und bienenverträglich erwiesen hatte (RITTER, 1978 a). Einzeluntersuchungen der Bienen zeigten jedoch, dass die Wirkung mit der Jahreszeit und der Befallsstärke sehr stark streute (RuTTNER et al., 1980). Diese unterschiedliche Wirkung ist auch von anderen Akariziden bekannt (SMIRNov, 1978). Die Nachbehandlung mit einem Standardpräparat eignet sich daher nur für Untersuchungen, in denen die Wirksamkeit verschiedener Präparate miteinander verglichen wird. Es wurde daher ein Verfahren entwickelt, mit dem die verbliebenen Milben in kurzer Zeit bestimmt werden können.

Die behandelten Bienen aus den Laborversuchen werden grundsätzlich nach Versuchsende abgetötet. Behandelten Bienenvölkern kann eine Stichprobe von 800 bis 1000 Bienen entnommen werden. Hierzu werden die Bienen einer Wabe - wenn möglich einer Brutwabe - abgetötet. Eine vollständige Heilung der Völker kann aber nur festgestellt werden, wenn ebenfalls sämtliche Bienen abgetötet werden. Damit in den Waben eingeklemmte bereits durch die Behandlung abgetötete Milben das Untersuchungsergebnis nicht verfälschen, werden die Bienen vor der Abtötung in einen Schwarmkasten abgekehrt. Tote Milben, die durch das Bodensieb auf die Papiereinlage fallen, werden dem Behandlungserfolg und lebende dem Restmilbenbefall zugerechnet.

Jeweils etwa 1000 der abgetöteten Bienen werden in einem Erlenmeyerkolben (Inhalt 1 l) mit $400 \mathrm{ml} \mathrm{Waschbenzin} \mathrm{übergossen} \mathrm{und} 15 \mathrm{Min}$. eingeweicht. Eingefrorene Bienenproben werden zuvor aufgetaut. Anschliessend werden die Bienen in einem motorgetriebenen Rüttelgerät (SALvis) weitere 15 Min. ständig geschüttelt. In einer Wanne $(43 \times 33 \times 14 \mathrm{~cm})$ werden die vollständig von Waschbenzin bedeckten Bienen 
nochmals kurz geschüttelt (Abb. 4) und mit einem groben Sieb $(30 \times 25 \times 5 \mathrm{~cm})$ entfernt. Die Milben werden in einem Feinsieb (Mullstoff) aufgefangen und gezählt. Das Auswaschen mit Benzin sollte wegen der auftretenden Dämpfe und der ständigen Feuergefahr nur unter dem Abzug durchgeführt werden.

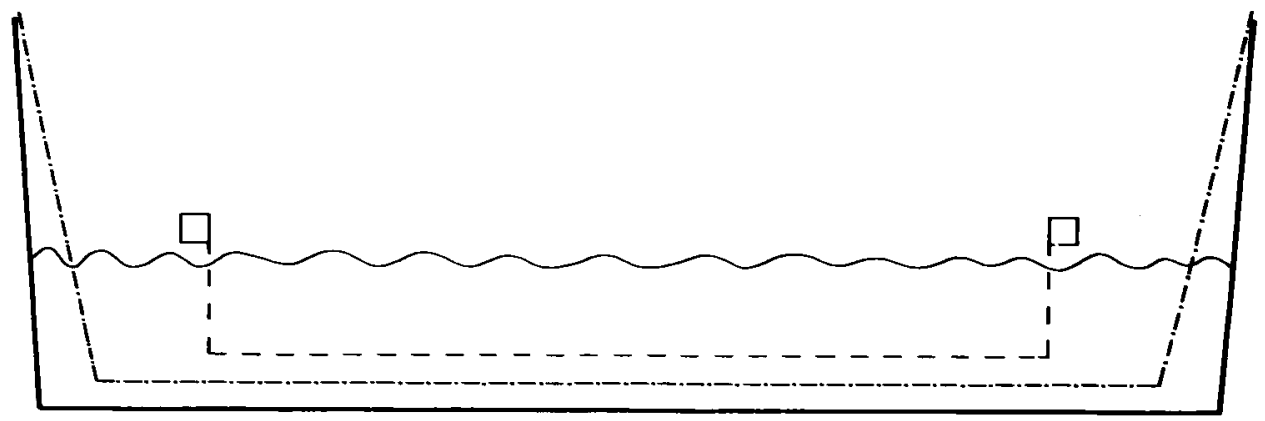

Авв. 4.-Querschnitt durch die Ausschütlelvorrichtung.

- $-\quad$ : grobes Sieb.

-... : feines Sieb.

: Wanne.

: Flüssigkeitsstand (Waschbenzin).

FIG. 4. - Coupe du dispositif pour secouer les abeilles

- $\quad$ : : tamis grossier.

et récupérer les acariens.

: : tamis fin.

— $\quad$ : baquet.

Eine individuelle mikroskopische Kontrolle der Einzelbienen aus "Schwärmen " mit 100 bis 1000 Bienen ergab, dass mit dieser Methode bei Verwendung von Benzin im Durchschnitt über $99 \%$ der auf den Bienen verbliebenen Milben ausgewaschen werden (Tabelle). Mit Pentan, Tetrachlorkohlenstoff, $98 \%$ igen Alkohol und Wasser ohne Zusätze können dagegen nur 48 bis $84 \%$ der Milben erfasst werden. Werden dem Wasser nach Woelke (1967) Detergentien zugesetzt ( $1 \mathrm{ml}$ Pril auf 31 Wasser) so werden im Durchschnitt $91 \%$ der Milben ausgewaschen. Nach MAUTZ (1979) ist das Wasser mit Detergentienzusatz ( $1 \mathrm{ml}$ Lavex auf $31 \mathrm{Wasser}$ ) dem Benzin gleichwertig. Er fand mit einer Benzinauswaschung im Anschluss an eine Untersuchung mit dem Wasser keine weiteren Milben. Über $70 \%$ der von MAUTZ untersuchten Proben (100 g Bienen) enthielten jedoch weniger als 2 Milben und maximal 5 Milben. Der Fehler der Methode nimmt mit zunehmender Milbenzahl in der Probe wohl zu. Entspanntes 
TAB. 1. - Ausschüttelergebnisse mit verschiedenen Waschmitteln.

TABL. 1. - Résultats du secouage dans divers produits de lavage.

\begin{tabular}{|c|c|c|c|c|c|c|}
\hline $\begin{array}{l}\text { Auswaschmittel } \\
\text { Produit de lavage }\end{array}$ & $\begin{array}{c}\text { Zahl } \\
\text { der Proben } \\
\text { Nombre } \\
\text { d'échantillons }\end{array}$ & $\begin{array}{l}\text { Zahl der Bienen } \\
\text { Pro Probe }(\varnothing) \\
\text { Nombre d'abeilles } \\
\text { par échantillon }(\varnothing)\end{array}$ & \multicolumn{2}{|c|}{$\begin{array}{c}\text { Gesamt-Milbenzahl } \\
\text { pro Probe }(\varnothing) \\
\text { Nombre total } \\
\text { d'acariens } \\
\text { par échantillon }(\varnothing)\end{array}$} & \multicolumn{2}{|c|}{$\begin{array}{c}\% \text { ausgewaschener } \\
\text { Milben }(\varnothing) \\
\text { \% d'acariens extraits } \\
\text { par lavage }\end{array}$} \\
\hline $\begin{array}{l}\text { Pentan } \\
\text { Pentane }\end{array}$ & 6 & 100 & 40 & $S=10,3$ & 83,8 & $S=18,6$ \\
\hline Tetrachlorkohlenstoff & 4 & 100 & 25 & $S=20,7$ & 73,4 & $S=19,7$ \\
\hline $\begin{array}{l}\text { Alkohol } 98 \% \\
\text { Alcool } 98 \%\end{array}$ & 4 & 100 & 34 & $S=22,4$ & 65,1 & $\mathrm{~S}=12,4$ \\
\hline $\begin{array}{l}\text { Wasser ohne Zusatz } \\
\text { Eau pure }\end{array}$ & 4 & 100 & 33 & $S=23,4$ & 48,0 & $\mathrm{~S}=33,0$ \\
\hline $\begin{array}{l}\text { Wasser mit Deter- } \\
\text { gentienzusatz } \\
\text { Eau + détergent }\end{array}$ & 6 & 100 & 79 & $S=31,9$ & 90,3 & $\mathrm{~S}=3,0$ \\
\hline $\begin{array}{l}\text { Wasser mit Deter- } \\
\text { gentienzusatz } \\
\text { Eau + détergent }\end{array}$ & 10 & 1000 & 113 & $S=47,1$ & 89,8 & $S=5,8$ \\
\hline $\begin{array}{l}\text { Waschbenzin } \\
\text { Essence }\end{array}$ & 10 & 100 & 53 & $\mathrm{~S}=18,7$ & 99,6 & $\mathrm{~S}=0,8$ \\
\hline $\begin{array}{l}\text { Waschbenzin } \\
\text { Essence }\end{array}$ & 10 & 600 & 283 & $\mathrm{~S}=181,9$ & 99,6 & $S=0,5$ \\
\hline $\begin{array}{l}\text { Waschbenzin } \\
\text { Essence }\end{array}$ & 6 & 1000 & 98 & $S=19,1$ & 99,3 & $S=1,1$ \\
\hline
\end{tabular}

Wasser ist nach unserer Erfahrung nur für eine grobe Schätzung des Milbenbefalls geeignet.

Das Verfahren kann für grössere Bienenproben weiter vereinfacht werden, wobei allerdings eine grössere Ungenauigkeit in Kauf genommen werden muss. Die 6000 bis 8000 Bienen werden in einem üblichen Eimer (101 Inhalt) mit Siebeinsatz $(2,6 \mathrm{~mm}$ Maschenweite) eine 1/2 Stunde unter ständigem Rühren eingeweicht.

Die durch das Sieb in den untergestellten Eimer fallenden Milben und Kleinpartikel können über ein Feinsieb (Mullbinde) abgefiltert werden. Die Untersuchung von 4 Proben mit durchschnittlich 7500 Bienen und 237 Milben ergab nach der anschliessenden Kontrolle im Labor, dass im Durchschnitt 90,0 \% $(\mathrm{s}=1,2)$ der Milben mit dieser Methode erfasst werden.

Die bisher gewonnenen Ergebnisse zeigen, dass eine Mittelprüfung ohne dieses Diagnoseverfahren ungenau ist und der hohe Bienenverschleiss daher verantwortet werden kann. Selbst benachbarte Völker sind häufig zu unterschiedlich befallen, um allein aus den nach der Behandlung abfallenden Milben auf die Wirkung eines Akarizids zu schliessen. 


\section{RÉSUMÉ}

L'homologation de médicaments efficaces dans le traitement de la varroose suppose un examen biologique détaillé (Fig. 1), qui commence avec les divers tests en laboratoire et en champ et se termine par l'expérimentation clinique dans des essais en champ. Des recherches sur les résidus et la toxicologie préparent le processus d'homologation.

\section{Recherches toxicologiques vis-à-vis de l'abeille}

Différentes doses de la substance étudiée ont été testées dans des cagettes renfermant environ 100 abeilles (Fig. 2). Les recherches au laboratoire ont été menées à la température constante de $20^{\circ} \mathrm{C}$ avec une humidité relative de $55 \%$. Deux traitements ont été appliqués aux abeilles : soit pulvérisation d'l $\mathrm{ml} \mathrm{du}$ produit sur les abeilles, soit évaporation de $2 \mathrm{ml}$ du produit dans des petites bouteilles munies d'une mèche et placées dans les cagettes. Afin de tester une action systémique le produit a été ajouté à la nourriture. On a procédé à 3 répétitions, avec 2 à 4 témoins, pour chacune des 4 doses de produit testé. En plus de la mortalité des abeilles on a déterminé la $\mathrm{DL}_{50}$.

\section{Recherches toxicologiques vis-ä-vis des acariens}

On a étudié la toxicité pour les acariens des doses non dangereuses pour les abeilles dans des groupes plus importants, d'environ 1500 à 2000 abeilles. Les acariens morts peuvent tomber à travers le fond de la caisse à essaim, muni d'une gaze (Fig. 3). On a vaporisé $4 \mathrm{ml}$ sur les abeilles ou bien on a traité l'une après l'autre 2 caisses à essaim dans une ruche vide par fumigation ou évaporation du produit. $\mathrm{La}^{\mathrm{L} \mathrm{D}_{50}}$ a été déterminée au bout de 24 heures pour 3 doses différentes à raison de 3 essais et 2 témoins par dose.

\section{Tests en plein air}

On a traité des nucléi (4 rayons occupés), puis des colonies entières soit par pulvérisation de $10 \mathrm{ml}$ sur chaque face de rayon, soit par fumigation ou évaporation du produit dans la ruche. On a répété 4 à 5 fois chaque essai.

\section{Essais en champ à grande échelle}

Les essais en champ ont été menés dans une région bien délimitée sur environ 100 à 200 colonies. Puisqu'on ne peut atteindre les acariens au sein du couvain sous les opercules, il faut d'abord débarrasser les colonies du couvain avant de traiter par pulvérisation ou fumigation. Par contre les préparations volatiles peuvent être utilisées sur une période de plus de 3 semaines. $10 \%$ des colonies ont été tuées pour contrôler le résultat du traitement.

\section{Diagnose}

a) Détermination de la mortalité des acariens dans la ruche.

Les acariens qui se sont détachés à la suite du traitement sont récoltés sur un support clair renforcé d'une gaze (maille de $2,6 \mathrm{~mm}$ ). Si les acariens ne sont que passagèrement endormis, il est nécessaire d'enduire le support d'un produit adhésif.

b) Détermination de l'incidence des acariens encore présents sur la colonie traitée.

La détermination des acariens encore présents sur les abeilles après le traitement est essentielle pour pouvoir juger les tests de laboratoire, les tests à l'extérieur à petite échelle et ceux à grande échelle.

- Incidence sur le couvain des acariens restants

On étudie les cellules individuellement par des échantillons pris au hasard ( 300 cellules) ou par des petites mesures de couvain. On porte en étuve $\left(34^{\circ} \mathrm{C}, 70 \%\right.$ d'humidité relative) les rayons à éclore et on extrait par lavage les abeilles mortes.

\section{- Incidence sur les abeilles adultes des acariens restants}

La détermination de l'incidence des acariens restants par un post-traitement avec un acaricide standard ne convient que pour comparer l'efficacité des diverses préparations. Les abeilles sont tuées d'après un nouveau procédé, mises à tremper pendant $15 \mathrm{mn}$ dans de l'essence et finalement secouées continuellement durant $15 \mathrm{mn}$. Dans un baquet on recouvre totalement les abeilles d'essence puis, après les avoir un peu secouées, on les retire à l'aide d'un tamis grossier et les acariens sont capturés avec un tamis fin (Fig. 4). 
Les contrôles des abeilles individuelles ont montré que cette méthode permettait d'extraire par lavage $99 \%$ des acariens. Les autres produits de lavage testés ont donné des pourcentages moindres (Tabl. 1).

Le test d'un produit sans ce procédé de diagnose est inexact. Le dégât èlevé causé aux abeilles peut ainsi se justifier.

\section{LITERATURNACHWEIS}

LANGE A. B., 1976. - Zitiert nach SMIRNOv, OIE/Apimondia-Seminar : Äthologie und Bekämpfung der Varroatose. Bukarest, Rumänien, S. 60-74.

MAUTZ D., 1979. - Untersuchungsergebnis : Erster bayerischer Varroa-Befall. Imkerfreund, 9 : 272-277.

Rutter W., 1978 a. - Gegenwärtiger Stand der Varroa-Bekämpfung im Tunusgebiet : Ergebnis der Varostan-Behandlung im Herbst 1977. OIE/Apimondia-Seminar über Bienenpathologie, Bukarest, Rumänien, S. 76-80.

RitTer W., 1978 b. - Diskussion auf dem OIE/Apimondia-Seminar über Bienenpathologie, Bukarest, Rumänien, S. 91.

RutTner F. (Federführend), 1980. - Allg. dische Imkerztg., Heft 5 (in Vorbereitung).

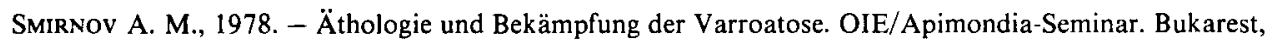
Rumänien, S. 60-74.

WoelKe O., 1967. - Milben-Wäscherei. Eine Methode zum Sammeln von Milben aus Fellen und Gefieder. Mikrokosmos, $3: 2$. 